\title{
OS DESASTRES NATURAIS NAS PERSPECTIVAS DO DESENVOLVIMENTO SUSTENTÁ VEL FORTE E FRACO
}

\author{
NATURAL DISASTERS FROM THE PERSPECTIVES OF STRONG AND WEAK \\ SUSTAINABLE DEVELOPMENT
}

\author{
Alex Santiago Nina ${ }^{1}$ \\ Ivonês Damasceno Lobo ${ }^{2}$
}

\section{PALAVRAS-CHAVE}

Desenvolvimento Sustentável Fraco. Desenvolvimento Sustentável forte. Desastres naturais.

KEYWORDS

Weak Sustainable Development. Strong Sustainable Development. Natural disasters.

\section{RESUMO}

Nascido com a proposta de compatibilização entre o crescimento econômico e preservação ambiental, o desenvolvimento sustentável possui duas correntes centrais - fraca e forte, as quais apresentam diferentes visões sobre os principais problemas globais, como superpopulação, escassez de recursos naturais, pobreza e desigualdade, degradação ambiental e mudanças climáticas. Estas escolas, no entanto, pouco têm explorado a temática dos desastres naturais, fenômenos com claros impactos socioeconômicos, cujas frequências vêm crescendo nos últimos anos, com relevância ainda maior no atual cenário de pandemia do novo coronavírus. Nesse sentido, o artigo tem como objetivo analisar a visão da sustentabilidade, nas suas correntes forte e fraca, a respeito da problemática dos desastres naturais. $\mathrm{O}$ estudo se baseia na pesquisa bibliográfica sobre desastres naturais e desenvolvimento sustentável. Os resultados apontam que a corrente fraca predomina na literatura internacional e tende a enxergar os desastres naturais como fatores externos ao desenvolvimento; enquanto a corrente forte predomina na literatura nacional e considera os eventos extremos como fatores intrínsecos ao desenvolvimento. $\mathrm{O}$ artigo propõe a conciliação entre as duas correntes, considerando a relação de feedback positivo entre desastres naturais e sustentabilidade.

\section{ABSTRACT}

Born with a proposal of compatibility between economic and environmental growth, or sustainable development, it has two central points - weak and strong, which shows different views on the main global problems, such as overpopulation, scarcity of natural resources, poverty and inequality, degradation environmental and climate change. These schools, however, have little explored the theme of natural disasters, phenomena with socioeconomic impacts, whose frequencies are increasing in recent years, with greater relevance in the current pandemic scenario of new coronaviruses. In this sense, the article aims to analyze the vision of sustainability, strong and weak, with respect to the issue of natural disasters. The study is based on bibliographic research about natural disasters and sustainable development. The results show that a weak current predominates in the international literature and tends to see natural

\footnotetext{
${ }^{1}$ Doutor em Ciências: Área de Concentração Desenvolvimento Socioambiental (UFPA). Docente da Universidade do Estado do Amapá (UEAP). Email: alex.0591@ @otmail.com

${ }^{2}$ Mestranda em Desenvolvimento Sustentável do Trópico Úmido (UFPA). Email: ivoneslobo@outlook.com
} 


\section{GESTÃO \& SUSTENTABILIDADE}

AMBIENTAL

\section{sunisul}

disasters as external factors to development; while the strong current predominates in the national literature and considers extreme events as intrinsic factors to development. The article offers a reconciliation between the two currents, considering a positive feedback relationship between natural disasters and sustainability.

\section{INTRODUÇÃO}

Os estudos sobre desenvolvimento têm suscitado uma série de linhas de pensamento nascidas a princípio nas ciências econômicas, mas que se ampliaram a partir da inserção de outras ciências como a ecologia. Os debates sobre a compatibilidade entre crescimento econômico e preservação ambiental culminaram com o advento do "desenvolvimento sustentável", um termo que passou a ter inúmeras controversas, resultando, ainda que de forma simplista e didática, em duas correntes: a sustentabilidade fraca e a sustentabilidade forte (DALY, 1997; FAUCHEUX, 1995; GEORGESCU-ROEGEN, 1979; HARTWICK, 2008; KESTEMONT, 2010, SOLOW, 1991; 1993; VIVIEN, 2005).

A perspectiva do desenvolvimento sustentável passou a nortear tanto políticas públicas como pesquisas científicas em diversos temas como pobreza, desigualdade, poluição atmosférica, mudanças climáticas, entre outros. Alguns exemplos recentes são: estudo da viabilidade da instalação de painéis solares e da gestão sustentável em instituições públicas (LIMA; ALMEIDA, 2021; SOARES; NADAE; NASCIMENTO, 2021); conhecimentos e práticas sustentáveis da agricultura familiar (NASCIMENTO et al., 2021); avaliação da efetividade de políticas de assentamento no que tange ao desenvolvimento sustentável (RIBEIRO et al., 2021); análise da contribuição científica para o entendimento das cidades sustentáveis (NEIVA; MARTIGNAGO, 2021).

São poucos os estudos, no entanto, que abordam a relação entre a sustentabilidade e os desastres naturais, fenômenos que claramente impactam a economia e o bem-estar social (BURBY et al., 2013; EL-MASRI; TIPPLE, 2002). Ressalta-se que, em decorrência das mudanças climáticas e do crescimento populacional, os desastres naturais estão se tornando cada vez mais frequentes no mundo. Recentemente, a pandemia do coronavírus, que pode ser considerada um tipo de desastre natural, já contaminou, até 17 de novembro de 2021, mais de 250 milhões de pessoas, com mais de 5 milhões de mortes (EUROPEAN CENTRE FOR DISEASE PREVENTION AND CONTROL - ECDC, 2021).

O presente artigo tem como objetivo analisar a visão da sustentabilidade, nas suas correntes forte e fraca, a respeito da problemática dos desastres naturais. A metodologia 


\section{GESTÃO \& SUSTENTABILIDADE} AMBIENTAL

\section{sunisul}

consistiu, primeiramente na síntese teórica da evolução dos termos "desenvolvimento" e “desenvolvimento sustentável” (Tópico 2); dos principais pontos de convergência e divergência entre as correntes da sustentabilidade forte e da sustentabilidade fraca (Tópico 3); e de conceitos básicos de desastres naturais. Em seguida, são apresentados: a metodologia, os resultados e as considerações finais.

\section{DO “DESENVOLVIMENTO” AO “DESENVOLVIMENTO SUSTENTÁVEL"}

Desde meados do século XX, o "desenvolvimento" tem sido entendido como o grande paradigma ou objetivo geral a ser alcançado por todas as sociedades. As raízes desta ideia remetem ao pensamento iluminista do final do século XVIII e início do século XIX, o qual preconizava a otimização dos processos produtivos, a razão, a liberdade de crítica, a noção de igualdade entre as pessoas, a oposição ao poder absoluto e o conhecimento como fonte de progresso (NAREDO, 2015; RÍO, 1997; SATRUSTEGUI, 2013).

Após a II Guerra Mundial, a economia neoclássica passou a entender o desenvolvimento como um processo linear, particularmente relacionado ao crescimento do Produto Interno Bruto (PIB) e consequente melhoria do bem-estar da população (GALBRAITH, 2007; MANKIW, 2009; SOLOW, 1991; 1993). Rostow (1974), adotando basicamente a produtividade como critério, considerava que as sociedades se encontravam em diferentes "etapas" de desenvolvimento ${ }^{3}$, que poderiam ser enquadradas em cinco categorias: a sociedade tradicional, as precondições para o arranco, o arranco, a marcha para a maturidade e a era do consumo de massa. Alguns autores alinhados a esse pensamento foram Kuznets e Murphy (1966), Lewis (1984) e Nurkse (1959).

A partir da década de 1960, no entanto, emergem uma série de críticas a noção de linearidade do desenvolvimento, pautadas na ideia de que a evolução da sociedade é complexa, não-linear e de difícil mensuração (FRANK, 2008; SEERS, 1971; SOARES JÚNIOR; QUINTELLA, 2008). As objeções recaiam principalmente ao uso do crescimento do PIB como sinônimo de desenvolvimento, alegando que esse indicador negligenciava fatores relevantes para o bem-estar social, tais como: a qualidade e o valor simbólico dos produtos, a produção voltada para o autoconsumo, a distribuição equitativa da riqueza produzida, o uso racional dos

\footnotetext{
${ }^{3}$ Além da produtividade, esse autor também elenca o nível de investimento e a renda média da população como variáveis importante para categorização das etapas do desenvolvimento, ambos possuindo um aspecto estritamente econômico (ROSTOW, 1974).
} 


\section{GESTÃO \& SUSTENTABILIDADE} AMBIENTAL

\section{DUnisul}

recursos naturais, os impactos ecológicos das atividades produtivas, a existência de limites socioambientais, a equidade de gênero, e o respeito à liberdade e aos direitos humanos (CAVALCANTI, 2010; GEORGESCU-ROEGEN, 1979; SATRUSTEGUI, 2013; SEN, 2000; SHIVA, 1988).

Alegava-se, ainda, que o discurso do "desenvolvimento" servia para ampliar as desigualdades sociais, conforme Veiga (2005, p. 79): “A renúncia à ideia de desenvolvimento deve-se ao fato de [ele] ter funcionado como armadilha ideológica inventada para perpetuar assimétricas relações entre as minorias dominantes e as maiorias dominadas, nos países e entre os países”. Dentre as críticas, destacaram-se, nas décadas de 1960 e 1970, uma série de estudos que indicavam a incompatibilidade do crescimento econômico global e a preservação do meio ambiente (CARSON, 1962; EHRLICH, 1970; MEADOWS et al., 1972).

A partir dos anos 1970, começou a se desenhar a noção de "desenvolvimento sustentável", como uma resposta a prerrogativa de impossibilidade de crescimento econômico com preservação ambiental, preocupando-se com a construção de estratégias de conciliação entre esses dois polos. A Primeira Conferência Mundial sobre o Homem e o Meio Ambiente, ocorrida em 1972 em Estocolmo, é considerada um marco político-conceitual para os debates mundiais entre preservação ambiental e crescimento econômico. É com o relatório de Brundtland de 1987, contudo, que surge o conceito mais difundido de desenvolvimento sustentável: "aquele que satisfaz as necessidades presentes, sem comprometer a capacidade das gerações futuras de suprir suas próprias necessidades” (BRUDTLAND, 1987, p.8).

Sobre o novo paradigma, entende-se o crescimento econômico como condição (e não sinônimo) para o desenvolvimento, o que mesmo assim tem suscitado críticas pósdesenvolvimentistas, como as de aproximação desta escola com a ideologia neoliberal (CROUCH, 2012; RIBEIRO, 2012; TULLOCH; NEILSON, 2014). Outro ponto de divergência advém da proposta, da escola sustentável, de política de conciliação entre diferentes atores sociais, particularmente o mercado, o governo e a sociedade civil. Na visão dos críticos, os conflitos entre esses atores são tão intensos ao ponto de ser impossível uma superação através de acordos consensuais (CAVALCANTI, 2010; GEORGESCU-ROEGEN, 1971; LEFF, 1993).

Por outro lado, as discussões sobre sustentabilidade englobam uma variedade de temas e pontos de vistas que, no geral, podem ser sistematizadas a partir da perspectiva de duas correntes político-científicas: a sustentabilidade fraca, nascida a partir da escola neoclássica; e a sustentabilidade forte, entendida como uma forma alternativa de desenvolvimento (KESTEMONT, 2010; MAYER, 2008; (NEUMAYER, 2003; VIVIEN, 2005). 


\section{FRACO VS. FORTE: UM DEBATE SOBRE SUSTENTABILIDADE}

Antes de adentrar nas diferenças de entendimento e estratégias das sustentabilidades fraca e forte, é necessário considerar alguns elementos em comum entre elas:

- Preocupam-se com a melhoria conjunta, bem como pelo uso operacional, de múltiplas dimensões do desenvolvimento, cujas básicas são a social, a ambiental e a econômica (ELKINGTON, 2001). Outras dimensões têm sido consideradas também relevantes, a exemplo da institucional, da cultural e da ética ${ }^{4}$ (PAULA; CAVALCANTI, 2000).

- Cada dimensão pode ser relativamente bem mensurada por diferentes tipos de capital (capital social, capital econômico e capital natural, por exemplo), de modo que o objetivo principal do desenvolvimento é o aumento desses capitais com o tempo, em virtude do progresso cultural, tecnológico, etc. (KESTEMONT, 2010);

- Preocupação com os impactos das atividades do presente ao bem-estar das futuras gerações, visando a equidade intergeracional; em particular o impacto cumulativo das atividades antrópicas ao meio ambiente (LENK; BESSAU, 2000);

- Visão sistêmica global, em múltiplas escalas, do particular ou local para o mundial. Uma ideia muito difundida tem sido a de "pensar global para agir local" (GASPARATOS; ELHARAM; HORNER, 2008);

- Reconhecimento de incertezas quanto as consequências das ações antrópicas e adoção do princípio da precaução, isto é, evitar as atividades cujos possíveis impactos negativos ainda são relativamente pouco conhecidos (LENK; BESSAU, 2000).

Apesar desses pontos em comum, existem diferenças essenciais entre 0 desenvolvimento sustentável fraco e forte (Figura 1), a começar pelos objetivos e pela forma como essas correntes interpretam as relações entre os diferentes capitais. A sustentabilidade fraca entende que o objetivo do desenvolvimento é o crescimento constante do capital total, ou seja, da soma dos capitais natural, social e econômico - pouco importando em qual desses se concentra o crescimento. Se uma dada sociedade teve que reduzir seu capital natural, através da degradação ambiental, por exemplo, para aumentar seu capital econômico, isto pode ser

\footnotetext{
${ }^{4}$ No presente estudo, se tratará das três dimensões básicas (natural, social e econômica), embora as ideias desenvolvidas possam ser ampliadas para as outras dimensões comumente reconhecidas na literatura.
} 


\section{GESTÃO \& SUSTENTABILIDADE}

AMBIENTAL

\section{ounisul}

benéfico caso o aumento do segundo seja, em termos absolutos, maior do que a redução do primeiro (HARTWICK, 2008; SOLOW, 1991; 1993; STIGLITZ, 1974; 1979). 


\section{sunisul उnime}

Figura 1 - Síntese das principais diferenças entre os modelos de desenvolvimento fraco e forte.

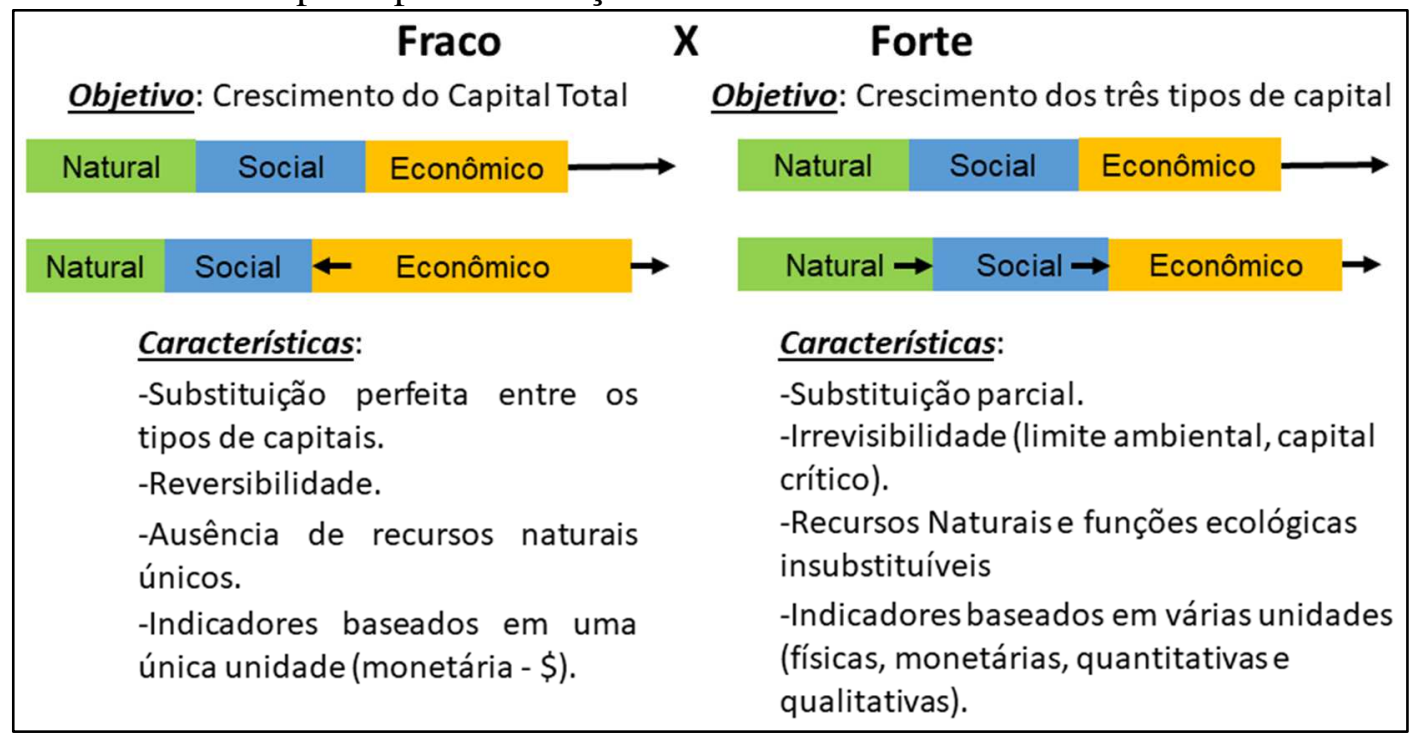

Fonte: Baseado em Kestemont (2010).

A ideia de que o importante é o capital total, e não as suas partes, advém da premissa de que é possível uma substituição perfeita entre os diferentes tipos de capitais, de modo que o ganho em capital econômico pode ser reconvertido integralmente em capital natural e vice-eversa. A corrente fraca acredita, portanto, na reversibilidade dos processos de degradação socioambiental. Outra premissa é de que não existe recurso natural único ou insubstituível, isto é, cuja finalidade de uso não pode ser suprida por outro recurso natural, o que minimiza o problema da escassez. Por fim, a sustentabilidade fraca entende que os três tipos de capitais podem ser contabilizados com base em um mesmo indicador (monetário, via regra), justificando então a substituição perfeita entre eles (LOMBORG, 2004; SOLOW, 1991; 1993; STIGLITZ, 1974; 1979).

Já a sustentabilidade forte acredita que o desenvolvimento só será alcançado através do crescimento concomitante dos três tipos de capitais, visto que, para esta corrente, o social e o ambiental não podem ser contabilizados de forma consistente por indicadores estritamente econômicos (DALY, 1997; UNITED NATIONS, 2003). Um típico exemplo são as críticas feitas a renda per capita como indicador de pobreza e bem-estar, uma vez que nem todos os bens e serviços associam-se à lógica do mercado e as pessoas possuem diferentes necessidades de autonomia, liberdade, acesso à educação, saneamento e meio ambiente ecologicamente equilibrado (ALKIRE; FOSTER, 2011; SEN, 2000; RAVALLION, 2011). 


\section{GESTÃO \& SUSTENTABILIDADE AMBIENTAL \\ sunisul}

Desta forma, considerando que os diferentes capitais se associam às diferentes dimensões do desenvolvimento, a sustentabilidade forte também acredita que não existe uma relação de substituição perfeita entre eles. Não se pode, por exemplo, reduzir o capital natural/social em detrimento do aumento do econômico, pois isto poderia trazer consequências irreversíveis. Nesta perspectiva, a sociedade e o meio ambiente poderiam não se recuperar de um processo de que atingisse um certo limite de degradação socioambiental, associada a um determinado valor de redução do capital (crítico), seja natural ou social (DALY, 1997; GEORGESCU-ROEGEN ,1979; UNITED NATIONS, 2003).

O debate sobre sustentabilidade fraca e forte é recorrente em diversas frentes: em campos científicos (Economia Ambiental x Economia Ecológica); na proposição de indicadores (Índices de Sustentabilidade Empresarial x Pegada Ecológica); entre diversos autores (Lomborg x Brown; Solow x Georgescu-Roegen; Stiglitz x Daly); nas discussões políticas em geral. Apesar disto, o debate pouco adentra na problemática dos desastres naturais.

\section{DESASTRES NATURAIS}

Os desastres naturais são fenômenos comumente reportados, no imaginário popular e midiático, como sinônimo de desgraça, fatalidade ou infortúnio, cuja causa relaciona-se a um nível sobrenatural, irracional ou divino. O próprio termo "natural", por vezes, é personificado, de modo que os desastres passam a ser vistos como produtos da "atuação maléfica da natureza" (MONTEIRO; PINHEIRO, 2012, p. 3). Cientificamente, porém, a crença na origem estritamente "natural" dos desastres já foi superada, pois, se considera que os danos que esses eventos provocam também dependem de condicionantes socioeconômicos como pobreza, ocupação desordenada do solo, infraestrutura precária, falta de sistemas adequados de saneamento e saúde, dentre outros (LAVELL; MASKREY, 2014; WORLD BANK, 2010).

Desta forma, os desastres naturais podem ser entendidos como fenômenos de origem natural que, ao atingirem uma região habitada por populações humanas, causam danos socioeconômicos capazes de interagir com o processo de desenvolvimento da região. Existem diferentes maneiras com que esta interação é interpretada: 1) interrupção, quando o desastre é visto como um evento externo que provoca um rompimento no processo de desenvolvimento; 2) consequência, quando o desastre é visto como um evento interno resultante da falta de desenvolvimento; 3) feedback positivo ou realimentação, em que o desastre é visto tanto como 


\section{GESTÃO \& SUSTENTABILIDADE} AMBIENTAL

\section{¿unisul}

causa quanto como consequência do baixo desenvolvimento (ALBALA-BERTRAND, 2014; AYSAN; LAVELL, 2014).

A sustentabilidade forte tende a adotar a Abordagem Social dos desastres naturais, que considera esses eventos como consequências da dinâmica social, isto é, o aumento do número de desastres é resultado dos problemas sociais existentes, como a desigualdade (econômica, socioespacial, étnica, de gênero etc.). Já a sustentabilidade fraca associa-se à Abordagem Técnica, que vê os desastres naturais como causa ou acentuação dos problemas sociais, sobre grande influência das mudanças climáticas (NINA; ALMEIDA; LOBO, 2021).

\section{METODOLOGIA}

A metodologia consistiu nas seguintes etapas:

a) Pesquisa bibliográfica na plataforma do google acadêmico, adotando-se os termos: "natural disasters and sustainability" e "desastres naturais e sustentabilidade".

b) Seleção de 8 publicações internacionais e 8 publicações nacionais, com inclusão, a partir destas, de outras publicações, com base nos critérios de frequência de citação e potencial de contribuição para discussão da temática.

c) Análise das publicações selecionadas e classificação das publicações quanto a associação com as correntes fraca e forte do desenvolvimento sustentável. Considerou-se que quando os desastres naturais são entendidos como fenômenos externos ao processo de desenvolvimento, predomina a visão da sustentabilidade fraca; já quando são enxergados como fenômenos intrínsecos ao processo de desenvolvimento, predomina a visão da sustentabilidade forte (NINA, 2016; NINA; ALMEIDA; LOBO, 2021).

d) Avaliação quanto ao grau de discussão a cerca do desenvolvimento sustentável feito pelos estudos levantados, considerando duas classes: estudos que introduzem um arcabouço teórico e estudos que não introduzem.

\section{RESULTADOS}

O Tabela 1 apresenta a síntese dos estudos analisados, com as suas principais características. Percebe-se que, dos 16 estudos levantados ( 8 internacionais e 8 nacionais), 9 se 


\section{GESTÃO \& SUSTENTABILIDADE AMBIENTAL}

\section{sunisul}

associam à corrente fraca do desenvolvimento sustentável e 7 à corrente forte; além disso, 9 introduzem um arcabouço do desenvolvimento sustentável, enquanto 7 não.

Tabela 1 - Lista dos estudos levantados com as principais características.

\begin{tabular}{|c|c|c|c|c|}
\hline Estudo & Abrangência & Natureza & $\begin{array}{c}\text { Corrente } \\
\text { Associada }\end{array}$ & $\begin{array}{c}\text { Introduz um arcabouço } \\
\text { teórico de } \\
\text { desenvolvimento } \\
\text { sustentável? }\end{array}$ \\
\hline $\begin{array}{c}\text { El-Masri e Tipple } \\
(2002)\end{array}$ & Internacional & Artigo & Forte & $\operatorname{Sim}$ \\
\hline $\begin{array}{c}\text { Ayala-Carcedo } \\
(2004)\end{array}$ & Internacional & $\begin{array}{l}\text { Capítulo de } \\
\text { Livro }\end{array}$ & Fraca & Não \\
\hline Pelling (2001) & Internacional & Artigo & Fraca & Não \\
\hline Peduzzi (2019) & Internacional & Artigo & Forte & Sim \\
\hline Wesener (2015) & Internacional & Artigo & Fraca & Não \\
\hline $\begin{array}{l}\text { Public Entity Risk } \\
\text { Institute (PERI, } \\
\text { 2001) }\end{array}$ & Internacional & Livro & Fraca & Sim \\
\hline Salkin (2008) & Internacional & Artigo & Fraca & Não \\
\hline $\begin{array}{c}\text { Fernandez e } \\
\text { Caecero-Moreno } \\
(2021) \\
\end{array}$ & Internacional & Artigo & Fraca & Sim \\
\hline Nina (2016) & Nacional & $\begin{array}{l}\text { Dissertação de } \\
\text { Mestrado }\end{array}$ & Fraca & Sim \\
\hline Silva (2012) & Nacional & $\begin{array}{c}\text { Boletim } \\
\text { Legislativo }\end{array}$ & Fraca & Não \\
\hline $\begin{array}{c}\text { Bodstein e Barros } \\
(2012)\end{array}$ & Nacional & $\begin{array}{l}\text { Capítulo de } \\
\text { Livro }\end{array}$ & Forte & Não \\
\hline $\begin{array}{c}\text { Alves e Ribeiro } \\
\text { (2015) }\end{array}$ & Nacional & Artigo & Forte & Sim \\
\hline Coutinho (2016) & Nacional & Artigo & Forte & Sim \\
\hline Sena et al. (2016) & Nacional & Artigo & Forte & Sim \\
\hline Marques (2020) & Nacional & $\begin{array}{l}\text { Capítulo de } \\
\text { Livro }\end{array}$ & Forte & Sim \\
\hline $\begin{array}{l}\text { Nina, Almeida e } \\
\text { Lobo (2021) }\end{array}$ & Nacional & Artigo & Fraca & Não \\
\hline
\end{tabular}

Fonte: compilado pelos autores.

Considerando apenas os 8 estudos internacionais, 6 associam-se a corrente fraca e, entre esses, 4 não introduzem um arcabouço teórico de desenvolvimento sustentável; ao passo que os outros dois estudos internacionais associados a corrente forte introduzem tal arcabouço. Já 


\section{GESTÃO \& SUSTENTABILIDADE} AMBIENTAL

\section{sunisul उnims}

entre os 8 estudos nacionais, 5 associam-se a corrente forte e, desses, 4 apresentam um arcabouço do desenvolvimento sustentável, ao passo que entre os 3 associados à corrente forte, apenas 1 apresenta esse arcabouço.

Desta forma, os dados apontam que estudos internacionais tendem a se associar à corrente fraca, apresentando ou não um arcabouço sobre desenvolvimento sustentável. Já os estudos nacionais tendem a se associar à corrente forte e apresentar um arcabouço a cerca do desenvolvimento sustentável.

\subsection{DESASTRES NATURAIS E A SUSTENTABILIDADE FRACA}

A ideia do desastre natural como um fenômeno externo ao desenvolvimento associa-se à corrente fraca e advém da economia neoclássica, em particular do modelo de crescimento econômico estacionário de Robert Solow (1956), cuja principal característica é a trajetória de equilíbrio relativamente estável, ao longo prazo, mesmo sem a intervenção direta do governo. O modelo admite que: o crescimento econômico é independente de fatores exógenos; as políticas econômicas pouco interferem em sua tendência; existe uma convergência econômica entre países pobres e países ricos, no que se refere ao nível de capital, produto e consumo; e, após um choque (ex. crise financeira, guerra, desastre natural), a economia tem a capacidade de se recuperar.

Recentemente, Lee e Tang (2019), aplicando o teste de Granger para dados de 1974 a 2014 das Filipinas, concluíram que são os desastres naturais que impactam o desenvolvimento do PIB (e não o contrário), além de impactarem diretamente o mercado de crédito e indiretamente a manutenção da pobreza absoluta na região. De fato, nos estudos associados a corrente fraca, predominam os métodos quantitativos de análise dos desastres naturais.

Na literatura internacional, por exemplo, Ayala-Carcedo (2004) faz um diagnóstico, baseado em dados estatísticos, da evolução do número, tamanho, danos humanos e prejuízos de desastres naturais no mundo, considerando esses eventos como externos ao desenvolvimento. Embora o autor não faça uma discussão densa sobre sustentabilidade, há uma separação dos danos entre sociais e econômicos.

Pelling (2001) estudou as atividades de mitigação em várias cidades do Caribe e América Latina. A análise, majoritariamente, considera que tais atividades estão associadas a medidas de combate às "ameaças" naturais externas, tais como ações táticas de Defesa Civil 


\section{GESTÃO \& SUSTENTABILIDADE AMBIENTAL}

\section{sunisul}

(Guiana), atuação de Organizações Não Governamentais (ONGs) (Bridgetown - Barbados) e planejamento urbano (São Domingos - República Dominicana).

Wesener (2015) estudou a formação de espaços abertos iniciados por comunidades após a ocorrência de um terremoto na cidade de Christchurch (Nova Zelândia), relatando como um caso de sucesso em comparação com outras cidades também atingidas por desastres. Apesar disso, não há uma discussão densa a respeito do desenvolvimento sustentável, ao passo que os desastres são visualizados como fenômenos externos ao desenvolvimento.

Salkin (2008), estudando o plano de mitigação a desastres naturais de governos locais nos Estados Unidos, apontou para a necessidade de integração intergovernamental. Os links com as discussões a respeito de desenvolvimento sustentável também são poucos explorados, prevalecendo a ideia dos desastres naturais como fenômenos externos, típica da sustentabilidade fraca.

Fernandez e Caecero-Moreno (2021) fazem uma simulação, baseada em gamificação, considerando o designer de uma cidade sustentável (em termos de produção energética, educação, sistema de saúde, gestão de lixo, etc.) e seus efeitos ao aumento/redução de riscos naturais. Os desastres naturais são tratados, a partir da perspectiva dos hazards, como variáveis independentes de componentes da sustentabilidade urbana.

Um guia clássico, feito Public Entity Risk Institute (PERI, 2001), propõe uma série de ideias para a recuperação local pós-desastres pautadas na sustentabilidade. A obra se dedica a discussão teórica do desenvolvimento sustentável, debruçando, posteriormente, a explorar seis princípios para a recuperação pós-desastres sustentável: processo participativo, qualidade de vida, vitalidade econômica, equidade social e intergeracional, e resiliência. $O$ foco na recuperação faz com que predomine na obra a noção do desastre natural como um fenômeno externo ao desenvolvimento.

Entre os estudos nacionais, Nina (2016), avaliando os impactos das inundações de 2009 na Amazônia, a partir de um arcabouço teórico do desenvolvimento sustentável, considera que apenas alguns desastres (particularmente as inundações bruscas) impactam negativamente o desenvolvimento sustentável da região.

Em outro estudo, o mesmo autor, com colaboradores (NINA; ALMEIDA; LOBO, 2021), faz uma análise dos Bancos de dados S2ID (Sistema Integrado de Informações sobre Desastres) e EM-DAT (Emergency Disaster Database) para verificar o número de registro e as 


\section{GESTÃO \& SUSTENTABILIDADE} AMBIENTAL

\section{sunisul}

tendências de crescimento de ambos. Os autores encontraram diferenças significativas: o S2ID tem maior média de número de registros, enquanto o EM-DAT tem menor crescimento anual do número de desastres naturais, com tendência a estabilizar. $O$ estudo conclui que a quantificação dos desastres naturais, mesmo que com limitações, é benéfica à gestão de riscos naturais, associando-se à corrente fraca do desenvolvimento sustentável.

Em outro estudo nacional, Silva (2012) faz uma reflexão sobre "Desastres Naturais e Desenvolvimento Sustentável”, onde aborda a Estratégia Internacional para Redução de Desastres (EIRD), destacando: perigos naturais, vulnerabilidade e risco. O autor faz uma descrição temporal da evolução da preocupação em torno dos riscos de ocorrência de desastres e seu impacto ao meio ambiente e aos seres humanos. Na década de 1970, o objetivo era buscar respostas às ocorrências extremas de desastres para atenuar os impactos aos atingidos. Em 1994 criou-se a primeira estratégia, juntamente ao primeiro plano de ação para reduzir os riscos, concomitantemente a implementação do Desenvolvimento Sustentável, ao nível global, voltado à vulnerabilidade, avaliação e gestão de riscos de desastres. Em 2005, a Organizações das Nações Unidas (ONU) apresentou o Marco de Ação de Yogo, visando tornar as nações e comunidades vulneráveis mais resilientes. O autor destaca a importância da Defesa Civil na consolidação do gerenciamento de riscos e chama atenção para a cultura comportamental das pessoas em relação aos riscos e à preservação do meio ambiente. Também ressalta a importância do poder público no que tange ao desenvolvimento de políticas que atendam às demandas de proteção e reconstrução de áreas atingidas, além de oferecer um ambiente seguro às pessoas que se encontram em áreas de vulnerabilidades. A perspectiva de hazards (ameaças externas) prevalece.

\subsection{DESASTRES NATURAIS E SUSTENTABILIDADE FORTE}

A corrente da sustentabilidade forte, no geral, usa métodos qualitativos de análise dos desastres naturais, visualizando-os como fenômenos estritamente sociais, internos ao desenvolvimento e relacionadas com a segregação socioespacial. A valorização imobiliária, por exemplo, induziria os estratos mais pobres da sociedade a residir em locais mais expostos às ameaças naturais, configurando o que Acselrad (2006) denomina "desigualdade ambiental". Desta forma, a corrente fraca enxerga que os benefícios das medidas de combate aos desastres naturais só poderão ocorrer de forma efetiva através do aumento da qualidade da governança e 


\section{GESTÃO \& SUSTENTABILIDADE} AMBIENTAL

\section{sunisul}

da participação popular no processo de estabelecimento de estratégias (UNITED NATIONS, 2015), bem como da redução das desigualdades sociais (VALENCIO, 2014).

Entre os estudos internacionais levantados, apenas o de El-Masri e Tiplle (2002) e o de Peduzzi (2019) seguiram esta corrente. Para El-Masri e Tipple (2002), os desastres naturais devem ser considerados como um problema não resolvido de desenvolvimento e não como um evento imprevisível, isolado ou independente. A mitigação dos desastres naturais deve combater os processos socioeconômicos que marginalizam as pessoas e aumentam a vulnerabilidade.

Já Peduzzi (2019) considera que temas que suscitaram a emergência histórica do conceito de desenvolvimento sustentável possuem estritas relações com a gestão de riscos naturais. $\mathrm{O}$ crescimento populacional acentua a exposição, uma vez que aumenta o número de pessoas morando em áreas perigosas, ao passo que o crescimento econômico acentua a vulnerabilidade econômica aos desastres naturais. $\mathrm{O}$ autor considera que embora predomine, na mídia e em boa parte da literatura, a ideia de desastres naturais como eventos súbitos, os riscos a eles associados são construídos paulatinamente, à medida que as vulnerabilidades sociais se desenvolvem.

Entre os estudos nacionais, Marques (2020) aborda a importância do debate sobre a resiliência urbana e o papel dos Objetivos do Desenvolvimento Sustentáveis (ODS) para o bemestar populacional. O autor destaca que os desastres naturais, embora considerados fenômenos naturais, apresentam uma construção social ocasionado pela dinâmica multidimensional e escalar, destacando o papel do governo e das instituições. O autor trata ainda sobre a governança urbana global, estreitando sua reflexão no âmbito brasileiro, onde não se tem uma mensuração adequada dos indicadores de resiliência em relação à realidade apresentada no país.

Bodstein e Barros (2012) buscaram evidenciar os principais problemas da má gestão pública, capazes de interferir negativamente na adoção do desenvolvimento sustentável - dentre eles os desastres naturais, dos quais se destacaram o gerenciamento do processo de prevenção e enfrentamento aos riscos naturais. Os autores chamam atenção para a necessidade de mudança cultural, seja da população, seja dos órgãos responsáveis pela urbanização, em relação ao crescimento exacerbado e ocupação de áreas de riscos,

Alves e Ribeiro (2015) mostraram a importância da Lei n. 12.608/2012 (Política Nacional de Proteção e Defesa Civil - PNPDEC) na gestão de desastres naturais e na implementação da sustentabilidade em áreas de riscos. Essa lei tem papel fundamental na 


\section{GESTÃO \& SUSTENTABILIDADE AMBIENTAL}

\section{sunisul}

promoção da sustentabilidade em áreas vulneráveis por meio de iniciativas ambientais, econômicas e sociais, bem como nas realocações da população, que se encontram em riscos, para áreas seguras e, desta forma, construindo cidades sustentáveis e resilientes.

Coutinho (2016) aborda a importância do direito de proteção aos danos de desastres, das políticas públicas voltadas às áreas atingidas, da proteção do meio ambiente e do papel do Estatuto da Cidade mediante as questões ambientais. $\mathrm{O}$ autor defende que a prática da sustentabilidade, no âmbito do desenvolvimento, é um direito fundamental a ser exercido, principalmente por estar associada à política urbana.

Sena et al. (2016) fez uma revisão dos Objetivos do Desenvolvimento Sustentável (ODS), analisando índices de desigualdades sociais, econômicas e ambientais. Tal análise, feita especialmente para municípios do semiárido brasileiro em relação aos municípios do país, apontou para as influências negativas das secas aos ODS.

\subsection{O FEEDBACK POSTIVO ENTRE DESASTRES NATURAIS E SUSTENTABILIDADE}

O entendimento dos desastres naturais como fenômenos internos ou externos ao desenvolvimento sustentável pode encontrar uma terceira opção: a relação de feedback positivo entre esses fenômenos, de modo que desastres tendam a impactar negativamente o desenvolvimento e o pouco desenvolvimento tenda a aumentar a vulnerabilidade aos desastres naturais. Nina (2016), por exemplo, estudando o impacto das inundações de 2009 ao crescimento econômico de municípios da Amazônia, considera que o fato dos danos, tanto em termos absolutos como relativos, serem maiores nos municípios mais ricos indica que o crescimento econômico dos municípios não está sendo acompanhado de um desenvolvimento em termos qualitativos. $\mathrm{O}$ autor admite que se um evento natural provoca consequências, positivas ou negativas, ao PIB, isto inevitavelmente provocará impactos econômicos, sociais e/ou ambientais. A Tabela 2 apresenta um exemplo hipotético dessa situação para o caso de inundações.

Tabela 2 - Exemplos hipotéticos de impactos econômicos, sociais e ambientais decorrentes de inundações com consequências negativas e positivas ao PIB.

\begin{tabular}{|c|c|c|c|}
\hline \multirow{2}{*}{\multicolumn{2}{|c|}{ Impacto }} & \multicolumn{2}{|c|}{ Consequências no PIB } \\
\hline & & Negativas & Positivas \\
\hline Econômico & $\begin{array}{c}\text { Destruição da } \\
\text { infraestrutura } \\
\text { pública, comercial. }\end{array}$ & $\begin{array}{lr}\text { Perda do capital físico e } \\
\text { aumento } \\
\text { desemprego, }\end{array}$ & \begin{tabular}{|lr} 
Aumento da demanda para a & civil; \\
construção & e \\
Replanejamento & reordenamento das rogioss
\end{tabular} \\
\hline
\end{tabular}




\section{GESTÃO \& SUSTENTABILIDADE} AMBIENTAL

\section{sunisul}

\begin{tabular}{c|c|l|l} 
& $\begin{array}{l}\text { consequente redução da } \\
\text { produtividade. }\end{array}$ & $\begin{array}{l}\text { atingidas; Estímulo às } \\
\text { inovações tecnológicas. }\end{array}$ \\
\hline Social & $\begin{array}{c}\text { Pessoas mortas, } \\
\text { feridas, } \\
\text { desabrigadas. }\end{array}$ & $\begin{array}{l}\text { Perda de capital humano } \\
\text { e aumento dos custos } \\
\text { com saúde }\end{array}$ & $\begin{array}{l}\text { Estímulo ao capital social (ex. } \\
\text { estabelecimento de laços de } \\
\text { solidariedade no combate à } \\
\text { inundação). }\end{array}$ \\
\hline Ambiental & $\begin{array}{c}\text { Transbordamento de } \\
\text { água em terras } \\
\text { produtivas. }\end{array}$ & $\begin{array}{l}\text { Aumento da erosão com } \\
\text { redução da capacidade } \\
\text { produtiva agropecuária. }\end{array}$ & Aumento da fertilidade do solo. \\
\hline
\end{tabular}

Fonte: Baseado em Nina (2016) e Hochrainer (2009).

Para a sustentabilidade fraca, o crescimento do capital econômico poderá facilmente ser utilizado para combater desequilíbrios provocados pela desigualdade ambiental. Num modelo análogo à curva de Kuznets ambiental, Lomborg (2004) considera que o crescimento do PIB dos países em desenvolvimento tem como consequência a redução dos danos provenientes de desastres naturais, em virtude do aumento da renda e do maior investimento em obras preventivas (ex. barragens de contenção de cheias, habitações resistentes, sistemas de alerta informatizados e eficientes).

A comparação dos danos humanos provocados por desastres naturais, entre países ricos e pobres, mostra que os primeiros podem prevenir ou mitigar os impactos de maneira mais eficiente e menos custosa em relação ao tamanho de suas economias (KAHN, 2005). Para Karim e Noy (2016), o principal motivo para esta diferença advém do fato de que, por possuir uma renda maior, tanto as populações como os governos dos países ricos têm mais recursos (em termos absolutos) para prevenção e mitigação de desastres naturais. Segundo os autores, a maioria dos estudos empíricos mostra que o aumento da renda per capita reduz os impactos negativos provocados por desastres naturais.

$\mathrm{Na}$ visão da sustentabilidade forte, embora tais efeitos do crescimento da renda não possam ser negligenciados, eles por si só não garantem uma redução dos danos provocados pelos desastres naturais ao longo prazo (MEYER et al., 2013). Em muitas situações, existe um tradeoff entre o crescimento econômico e a adoção de práticas de mitigação, sendo necessária a realização de análises de custo-benefício para verificar qual das duas opções trarão melhores retornos socioeconômicos (STERN, 2009).

São comuns, por exemplo, estudos que avaliam a viabilidade socioeconômica de obras preventivas ou de realocação da população residente em áreas perigosas (BÜCHELE et al., 


\section{GESTÃO \& SUSTENTABILIDADE} AMBIENTAL

\section{১unisul}

2006; KRON et al., 2012; MERZ et al., 2013). Tais estudos, geralmente, constatam que as práticas de prevenção aos desastres naturais trazem maiores benefícios tanto ao crescimento econômico como à redução dos danos humanos ao longo prazo.

Um elo comum entre as visões da sustentabilidade fraca e forte a respeito dos desastres naturais é a ideia da pobreza como fator determinante aos impactos dos desastres naturais mesmo que a corrente fraca enfatize mais a pobreza relativa (desigualdade) e a corrente forte a pobreza absoluta (econômica) (NINA, 2021).

A compreensão das relações entre desastres naturais e desenvolvimento sustentável é cada vez mais imperativa, sobretudo no contexto atual de pandemia da Covid-19 e de aceleração das mudanças climáticas. Estudos apontam que, ao nível global, os impactos negativos da Covid-19 foram maiores nos países e pessoas mais pobres, superando, nesta parcela da população, os efeitos negativos da crise de 2008 (DECERF et al., 2021; LABORDE; MARTIN, 2021).

No Brasil, evidências empíricas também sugerem que os efeitos negativos da Covid-19 são maiores aos mais pobres (NEVES et al., 2021; TAVARES; BETTI, 2021), com maior sensibilidade a indicadores de pobreza múltipla, como o Índice de Desenvolvimento Humano (IDH), que considera a privação de renda, saúde e educação (NINA et al., 2021). Esses estudos também evidenciam que as subnotificações, tanto de casos como de mortes, são maiores nas regiões menos desenvolvidas, em conformidade com o problema do "desastre silencioso", comumente reportando na literatura de desastres naturais. Esse problema ocorre quando as autoridades públicas não têm capacidade institucional de registrar, de forma adequada, as situações de desastres naturais (NINA; ALMEIDA; LOBO, 2021; VENTURATOLANDMAN; VALENCIO, 2014).

Quanto as mudanças climáticas, o mais recente estudo do Intergovermental Panel on Climate Change (IPCC, 2021) considera irrefutável a hipótese de que as ações antrópicas provocam as mudanças do clima, cujos efeitos já estão sendo vivenciados e tendem a se acentuar no futuro. Vale desatacar que o crescimento do número de ocorrências, danos e prejuízos proporcionados pelos desastres naturais no século $\mathrm{XX}$ ocorreu majoritariamente nos grupos de desastres climáticos, como secas, inundações, tempestades, dentre outros (LEANING; GUHA-SAPIR, 2013). No Brasil, os desastres naturais historicamente mais frequentes também são os climáticos, embora com natureza diferenciada nas diferentes regiões do país (predominando inundações no Norte, deslizamentos no Sudeste, secas no Nordeste, 


\section{GESTÃO \& SUSTENTABILIDADE}

AMBIENTAL

\section{sunisul}

queimada no Centro-Oeste e frentes frias no Sul) (TOMINAGA; SANTORO; AMARAL, 2009). Os cenários de mudanças climáticas tendem a acentuar esses eventos extremos (MARENGO et al., 2011).

\section{CONCLUSÕES}

O desencadeamento histórico das diversas ideias sobre desenvolvimento sustentável foi fundamental para as diferentes percepções dos desastres naturais. Os resultados da pesquisa realizada apontam que predomina, na literatura internacional, a visão da sustentabilidade fraca sobre os desastres naturais; ao passo que, na literatura nacional, predomina a visão da sustentabilidade forte, sendo mais frequente a introdução de um arcabouço teórico a respeito do desenvolvimento sustentável.

Na sustentabilidade fraca, os desastres são vistos como fenômenos externos, capazes de provocar uma interrupção no processo de desenvolvimento. Tal interrupção, no entanto, tende a ser momentânea e possuir pouco impacto ao longo prazo - embora alguns estudos sugerem o contrário. Ao separar os desastres naturais do processo de desenvolvimento, tal visão tem como principal vantagem a aplicabilidade de métodos quantitativos de avaliação de impactos e riscos, podendo servir de base a definição de políticas institucionais de combate a esses eventos. Essa ideia, no entanto, tem sido criticada pelos defensores da sustentabilidade forte, que a consideram como limitante, à medida que não reflete sobre os processos sociais de construção dos riscos naturais.

A sustentabilidade forte entende que os desastres naturais são construtos sociais, particularmente associados a questão da desigualdade nas suas mais diversas dimensões (econômica, cultural, territorial), havendo maior necessidade de atuação do poder público para resolução desse problema-raiz. Sua principal vantagem está na visão mais holística das questões sociais inerentes às ocorrências de desastres naturais. Sua desvantagem está no rechaço ao uso dos métodos quantitativos, o que, na visão dos defensores da sustentabilidade fraca, dificulta um olhar mais pragmático para o problema.

Para minimização dos conflitos entre as duas correntes, o presente estudo sugere uma terceira visão: a relação de realimentação (feedback positivo) entre ameaças naturais e vulnerabilidade socioeconômicas. Não há como negar a importância da quantificação dos desastres (em número de ocorrência, magnitude, intensidade, etc.) para boa gestão de riscos 


\section{GESTÃO \& SUSTENTABILIDADE} AMBIENTAL

\section{¿unisul}

naturais. Da mesma forma, não há como negar também a importância da compreensão desse fenômeno como uma construção social, sobretudo relacionada as condições de pobreza e vulnerabilidade.

\section{REFERÊNCIAS}

ACSELRAD, H. Vulnerabilidade ambiental, processos e relações. In: ENCONTRO NACIONAL DE PRODUTORES E USUÁRIO DE INFORMAÇÕES SOCIAIS, ECONÔMICAS E TERRITORIAIS, 2. 2006. Rio de Janeiro. Anais... Rio de Janeiro: FIBGE, 2006, p. 1-5.

ALBALA-BERTRAND, J. Disasters and the Networked Economy: A book summary. Working Paper Queen Mary, University of London, n. 718, p. 1-41, abr. 2014.

ALKIRE, S.; FOSTER, J. Counting and Multidimensional Poverty Measurement, Journal of Public Economics, v. 95, n. 7-8, p. 476-487, 2011.

ALVES, H. R.; RIBEIRO, J. C. J. A política nacional de proteção e defesa civil e a busca pela sustentabilidade nas áreas vulneráveis dos centros urbanos. Revista do Direito, v. 2, n. 46, p. 63-85, 2015.

AYALA-CARCEDO, F. A risk analysis and sustainability approach to natural disaster assessment and mitigation philosophy in the Word. In: HEIDELBERG. Natural Disasters and Sustainable Development. Berlin: Springer, 2004. p. 271-295.

AYSAN, Y.; LAVELL, A. Disaster risk governance during the HFA implementation period, New York: UNDP, 2014. 57p.

BODSTEIN, A.; BARROS, A. M. A. Gestão de riscos no contexto do desenvolvimento sustentável. In: PHILIPPI, A; SAMPAIO, C; FERNANDES, V. Gestão de Natureza Pública e Sustentabilidade. São Paulo: Editora Manolo, 2012.

BRASIL. Lei 12.608, de 10 de abril de 2012. Institui a Política Nacional de Proteção e Defesa Civil - PNPDEC; dispõe sobre o Sistema Nacional de Proteção e Defesa Civil - SINPDEC e o Conselho Nacional de Proteção e Defesa Civil - CONPDEC; autoriza a criação de sistema de informações e monitoramento de desastres; altera as Leis nos 12.340, de 1o de dezembro de 2010, 10.257, de 10 de julho de 2001, 6.766, de 19 de dezembro de 1979, 8.239, de 4 de outubro de 1991, e 9.394, de 20 de dezembro de 1996; e dá outras providências. Diário Oficial da União, 2012.

BRUNDTLAND, G. (Ed.). Report of the World Commission on Environment and Development: our common future. Norway: United Nations, 1987. 318p.

BÜCHELE, B.; KREIBICH, H.; KRON, A.; THIEKEN, A.; IHRINGER, J.; OBERLE, P.; MERZ, B.; NESTMANN, F. Flood risk mapping: contributions towards an enhanced assessment of extreme events and associated risks. Natural Hazards and Earth System Sciences, v.6, p.485-503, jun. 2006. 


\section{GESTÃO \& SUSTENTABILIDADE} AMBIENTAL

\section{¿Unisul}

BURBY, Raymond et al. (Ed.). Environmental management and governance: Intergovernmental approaches to hazards and sustainability. Abringdon: Routledge, 2013.

CARSON, R. Silent spring. Boston: Houghton Mifflin Harcourt, 1962, 169p.

CAVALCANTI, C. Concepções da economia ecológica: suas relações com a economia dominante e a economia ambiental. Estudos Avançados, v. 24, n. 68, p. 53-67. 2010.

COUTINHO, N. C. A. Direitos fundamentais e desenvolvimento sustentável: a importância da proteção contra desastres hidrológicos. Revista Eletrônica do Curso de Direito da UFSM, v. 11, n. 3, p. 915-930, 2016.

CROUCH, Colin. Sustainability, neoliberalism, and the moral quality of capitalism. Business and Professional Ethics Journal, v. 31, n. 2, p. 363-374, 2012.

DALY, H. E. Reply to Solow/Stiglitz. Ecological Economics, n, 22, p. 271-273, 1997.

ELKINGTON, J. Sustentabilidade: canibais com garfo e faca. São Paulo: Makron Books, 2001.

EL-MASRI, Souheil; TIPPLE, Graham. Natural disaster, mitigation and sustainability: the case of developing countries. International planning studies, v. 7, n. 2, p. 157-175, 2002.

ERHLICH, P. The population bomb: population control or race to oblivion? New York: SIERRA CLUB-BALLANTINE, 1970, 123p.

EUROPEAN CENTRE FOR DISEASE PREVENTION AND CONTROL. ECDC. Covid-19 Situation Dashboard. 2021. Disponível em: https://qap.ecdc.europa.eu/public/extensions/COVID-19/COVID-19.html\#global-overviewtab. Acesso em: 17 nov. 2021.

FAUCHEUX, S. Economie des ressources naturelles et de l'environnement. Armand Colin: Paris, 1995.

FERNÁNDEZ, P.; CEACERO-MORENO, M. Urban Sustainability and Natural Hazards Management; Designs Using Simulations. Sustainability. v. 13, n. 649, p. 1-26, jan. 2021.

FRANK, Andre Gunder. The underdevelopment of development. Revista Eletrónica Centro de Estudo de Opinión, n. 17, 2008.

GALBRAITH, John Kenneth. The new industrial state. Princeton: Princeton University Press, 2007. 576p.

GASPARATOS, A.; EL-HARAM, M.; HORNER, M. A critical review of reductionist approaches for assessing the progress towards sustainability. Environmental Impact Assessment Review, v. 28, n. 4-5, p. 286-311, 2008.

GeORGESCU-ROEgen, N. The Entropy Law and the Economic Process. Cambrige: Harvard University Press. 1971. 


\section{GESTÃO \& SUSTENTABILIDADE} AMBIENTAL

\section{sunisul}

GEORGESCU-ROEGEN, N. Comments on the papers by Daly and Stiglitz. Scarcity and growth reconsidered, p. 95-105, 1979.

HARTWICK, J. What would Solow say?. Journal of Natural Resources Policy Research, v. 1, n. 1, p. 91-96, 2008.

HOCHRAINER, S. Assessing the macroeconomic impacts of natural disasters: are there any? Policy Research Working Paper, n. 4968, p.1-43, jun. 2009.

IPCC. INTERGOVERMENTAL PANEL ON CLIMATE CHANGE. Climate Change 2021: The Physical Science Basis. Contribution of Working Group I to the Sixth Assessment Report of the Intergovernmental Panel on Climate Change. Cambridge: Cambridge University Press. 2021.

KAHN, M. The death toll from natural disasters: The role of income, geography, and institutions. Review of Economics and Statistics, n. 87, v. 2, p. 271-284, 2005.

KARIM, A.; NOY, I. Poverty and natural disasters: a qualitative survey of the empirical literature. The Singapure Economic Review, v. 61, n. 1, p. 1-36, 2016.

KESTEMONT, B. Les indicateurs de développement durable: fondements et applications. In: 2010, p. 19-82.

Les indicateurs de développement durable. Bélgica: Université Libre de Bruxelles,

KRON, W.; STEUER, M.; LÖW, P.; WIRTZ, A. How to deal properly with a natural catastrophe database - analysis of flood losses. Natural Hazards and Earth System Sciences, v. 12, p. 535-500, 2012.

KUZNETS, Simon; MURPHY, John Thomas. Modern economic growth: Rate, structure, and spread. New Haven: Yale University Press, 1966.

LAVELL, A.; MASKREY, A. The future of disaster risk management: an on-going discussion. Environmental Hazards, v. 13, n. 4, p. 267-280, 2014.

LEANING, J.; GUHA-SAPIR, D. Natural disasters, armed conflict, and public health. New England journal of medicine, v. 369, n. 19, p. 1836-1842, 2013.

LEE, CHI-YU; TANG, C. How do natural disasters influence the rate of poverty? Journal of Poverty, v. 23, n. 6, p. 478-486, 2019.

LEFF, E. Marxism and the environmental question: From the critical theory of production to an environmental rationality for sustainable development. Capitalism Nature Socialism, v. 4, n. 1, p. 44-66, 1993.

LENK, T.; BESSAU, D. Das Konzept des Sustainable Development. Universität Leipzig Arbeitspapier, n. 4, p. 1-11, 2000.

LEWIS, W. Arthur. The state of development theory. The American Economic Review, v. 74, n. 1, p. 1-10, 1984. 


\section{GESTÃO \& SUSTENTABILIDADE} AMBIENTAL

\section{¿unisul हु}

LIMA, N.; ALMEIDA, L. Gestão sustentável na administração pública: uma análise no Tribunal de Justiça do Tocantins. Revista Gestão \& Sustentabilidade Ambiental, v. 10, n. 1, p. 81-102, mai. 2021. Disponível em: http://www.portaldeperiodicos.unisul.br/index.php/gestao_ambiental/article/view/8613/5691. Acesso em: 22 set. 2021.

LOMBORG, B. L'ecologiste sceptique: le véritable état de la planète. Paris: Collection Documents, 2004. 620 p.

MANKIW, N. Introdução a economia. $5^{a}$ ed. São Paulo: Cengage Learning, 2009. 809p.

MARENGO, J.; NOBRE, C.; CHOU, S.; TOMASELLA, J.; SAMPAIO, G.; ALVES, L.; OBREGÓN, G.; SOARES, W.; BETTS, R.; KAY, G. Riscos das mudanças climáticas no Brasil: análise conjunta Brasil-Reino Unido sobre os impactos das mudanças climáticas e do desmatamento na Amazônia. São Paulo: Instituto Nacional de Pesquisas Espaciais; Met Office Hadley Centre. 2011. 56p.

MARQUES, C. Resiliência urbana e bem-estar no Brasil: reflexões a partir dos objetivos do Desenvolvimento Sustentável. In: MAGNONI JÚNIOR, L. et al. Redução do risco de desastres e a resiliência no meio rural e urbano. 2 ed. São Paulo: CPS, 2020. 865 p.

MAYER, A. L. Strengths and weaknesses of common sustainability indices for multidimensional systems. Environment international, v. 34, n. 2, p. 277-291, 2008.

MEADOWS, D.; MEADOWS, D.; RANDERS, J.; BEHRENS, W. The limits to growth: a report for the Club of Rome's project of the predicament of mankind. New York: Universe Books, 1972. 205p.

MERZ, B.; KREIBICH, H.; LALL, U. Multi-variate flood damage assessment: a tree-based data mining approach. Natural Hazards and Eath System Sciences, v.13, p.53-64, jan. 2013.

MEYER, V.; BECKER, N.; MARKANTONIS, V.; SCHWARZE, R.; BERGH, J.; BOUWER, L.; BUBECK, P.; CIAVOLA, P.; GENOVESE, E.; GREEN, C.; HALLEGATTE, S.; KREIBICH, H.; LEQUEUX, Q.; LOGAR, I.; PAPYRAKIS, E.; PFURTSCHELLER, C.; PUSSIN, J.; PRZYLUSKI, V.; THIEKEN, A.; VIAVATTENE, C. Review article: Assessing the costs of natural hazards - state of the art an knowledge gaps. Natural Hazards Earth System Science, n. 13, p. 1351-1373, 2013.

MONTEIRO, J.; PINHEIRO, D. O desastre natural como fenômeno induzido pela sociedade: abordagens teóricas e metodologias operacionais para identificação/mitigação de desastres naturais. Revista Geografia, v. 2, n. 1, p 1-9, 2012.

NAREDO, J. M. La economía en evolución: historia y perspectivas de las categorías básicas del pensamiento económico. Madri: Siglo XXI de España Editores, 2015.

NASCIMENTO, S.; NASCIMENTO, J.; HANKE, D.; ÁVILA, M.; SILVA, F. Gestão ambiental e agricultura familiar: um olhar sobre o município de Dom Pedrito-RS. Revista Gestão \& Sustentabilidade Ambiental, v. 9, n. 3, p. 480-499, jul-set. 2021. Disponível em: http://www.portaldeperiodicos.unisul.br/index.php/gestao_ambiental/article/view/8336/5394. Acesso em: 21 set. 2021. 


\section{GESTÃO \& SUSTENTABILIDADE AMBIENTAL}

\section{sunisul}

NEIVA, S.; MATIGNANO, G. Análise da contribuição científica no entendimento da criação de uma cidade sustentável: uma revisão sistemática da literatura e uma metassíntese qualitativa. Revista Gestão \& Sustentabilidade Ambiental, Florianópolis, v. 10, n. 1, p. 470-492, mai. 2021. Disponível em: http://www.portaldeperiodicos.unisul.br/index.php/gestao_ambiental/article/view/8400/5690. Acesso em: 21 set. 2021.

NEUMAYER, E. Weak versus strong sustainability: exploring the limits of two opposing paradigms. Cheltenham: Edward Elgar Publishing, 2003.

NINA, A. Desastres naturais e pobreza absoluta na Amazônia: uma análise quantitativa. 2021. 178f. Tese de doutorado, Programa de Pós-Graduação em Desenvolvimento Sustentável do Trópico Úmido, Núcleo de Altos Estudos Amazônicos, Universidade Federal do Pará, Belém, 2021.

NINA, A. Impacto de desastres naturais ao Produto Interno Bruto dos municípios e suas relações com o desenvolvimento: o caso das inundações de 2009 na Amazônia. 2016. 85f. Dissertação de Mestrado, Universidade Federal do Pará, Belém, 2016.

NINA, A. S.; ALMEIDA, O. T.; LOBO, I. D. Banco de dados sobre desastres naturais no Brasil: uma análise comparativa entre o EM-DAT E o S2ID. Cadernos de Estudos Sociais, v. 36, n. $1,2021$.

NINA, A.S.; LOBO, I.D.; NINA, A.M.S.; CÁO, L.B.; MELO, L.A. Análise econométrica de indicadores de Covid-19 e de pobreza no Brasil. In: CONGRESSO BRASILEIRO INTERDISCIPLINAR EM CIÊNCIA E TECNOLOGIA, 2. Evento online, Anais... Evento online: Comissão Organizadora. 2021. p. 1-4.

NURKSE, Ragnar. Notes on 'unbalanced growth'. Oxford Economic Papers, v. 11, n. 3, p. 295-297, 1959.

PAULA, G.O.; CAVALCANTI, R.N. Ethics: essence for sustainability. Journal of Cleaner Production, n. 8, p. 109-117, 2000.

PEDUZZI, P. The disaster risk, global change and sustainability nexus. Sustainability. v. 11, n. 957, p. 1-21, fev. 2019.

PELLING, M.; UITTO, J. Small island developing states: natural disaster vulnerability and global change. Environmental Hazards. v. 3, p. 49-62, 2002.

PUBLIC ENTITY RISK INSTITUTE. PERI. Holistic Disaster Recovery: Ideas for Building Local Sustainability After a Natural Disaster. Boulder, Colorado: PERI, 2001. 236p.

RAVALLION, M. On multidimensional indices of poverty. Journal of Economic Inequality, v. 9, n. 2, p. 235-248, 2011.

RIBEIRO, F. O paradigma ambiental na globalização neoliberal: da condição crítica ao protagonismo de mercado. Sociedade e Natureza, Uberlândia, ano 24, n. 2, mai-ago, p. 211 226. 2012. 


\section{GESTÃO \& SUSTENTABILIDADE AMBIENTAL}

\section{ounisul}

RIBEIRO, N.; SANTOS, L.; GRACIANO, M.; THOMSON, C.; BORSATTO, R. Os desafios da implementação de projetos de desenvolvimento sustentável no território rural Vale do Ribeira-SP. Revista Gestão \& Sustentabilidade Ambiental, Florianópolis, v. 10, n. 2, p. 105124, jun. 2021. Disponível em: http://www.portaldeperiodicos.unisul.br/index.php/gestao_ambiental/article/view/9685/5732. Acesso em: 21 set. 2021

RÍO, E. Modernidad y posmodernidad: Cuadernos de trabajo. Madri: Talasa Ediciones, 1997.

ROSTOW, W. As cinco etapas do desenvolvimento - um sumário. In: Etapas do desenvolvimento econômico, um manifesto não-comunista. Rio de Janeiro: Zahar Editores, 1974. p. 16-30

SALKIN, P. Sustainability at the Edge: The Opportunity and Responsibility of Local Governments to Most Effectively Plan for Natural Disaster Mitigation. Environmental Law Reporter. News \& Analysis, Washington, v. 38, p. 10158-10170, 2008.

SATRUSTEGUI, K. Desenvolvimento, subdesenvolvimento, mau-desenvolvimento e pósdesenvolvimento: um olhar transdisciplinar sobre o debate e suas implicações. Revista Perspectivas do Desenvolvimento, n.1, p. 34-69. 2013.

SEERS, D.; JOY, L. Development in a divided world. Harmondsworth: Peguin Books, 1971, $367 \mathrm{p}$.

SEN, A. Desenvolvimento como liberdade. $7^{\text {a }}$ ed. São Paulo: Companhia das Letras, 2000. 410p.

SENA, A. et al. Medindo o invisível: análise dos Objetivos de Desenvolvimento Sustentável em populações expostas à seca. Ciência \& Saúde Coletiva, v. 21, p. 671-684, 2016.

SHIVA, V. Staying alive: women, ecology and survival in India. New Delhi: Panchshila Park. 1988, 233p.

SILVA, C. H. R. T. Desastres naturais e desenvolvimento sustentável. Brasília: Senado Federal, 2012. 6 p.

SOARES JÚNIOR, J.S.; QUINTELLA, R.H. Instrumentalização do desenvolvimento: teorias, conceitos e indicadores. Organização \& Sociedade, v. 15, n. 45, p. 61-78, abr-jun, 2008.

SOARES, C.; NADAE, J.; NASCIMENTO, D. Análise da viabilidade econômica-financeira da energia solar fotovoltaica em uma instituição de ensino superior no Estado do Ceará. Revista Gestão \& Sustentabilidade Ambiental, Florianópolis, v. 10, n. 2, p. 84-104, jun. 2021. Disponível em: http://www.portaldeperiodicos.unisul.br/index.php/gestao_ambiental/article/view/9871/5719. Acesso em: 21 set. 2021.

SOLOW, R. A contribution to the theory of economic growth. Quarterly Journal of Economics, n. 70, p. 65-94, 1956.

SOLOW, R. An almost practical step toward sustainability. Resources Policy, set. 1993. 


\section{GESTÃO \& SUSTENTABILIDADE} AMBIENTAL

\section{Unisul}

SOLOW, Robert Merton. Sustainability: an economist's perspective. Falmouth, MA: Woods Hole Oceanographic Institution. 1991.

STERN, N. Clima è vera emergenza. Tradução: Elena Balzno. Itália: Francesco Brioschi Editore, 2009.

STIGLITZ, J. E. A neoclassical analysis of the economics of natural resources. In: SMITH, V. K. (ed). Scarcity and Growth Reconsidered. Baltimore, MD: Johns Hopkins University Press, 1979. P. 36-66.

STIGLITZ, J. E. Growth with exhaustive natural resources: efficient and natural growth paths. Symposium on the Economics of the Exhaustive Resources. Review of Economic Studies, v. 45, p. 123-137, 1974.

TOMINAGA, L.; SANTORO, J.; AMARAL, R. (org.). Desastres naturais: conhecer para prevenir. $1^{a}$ ed. São Paulo: Instituto Geológico. 2009. 196p.

TULLOCH, Lynley; NEILSON, David. The neoliberalisation of sustainability. Citizenship, Social and Economics Education, v. 13, n. 1, p. 26-38, 2014.

UNITED NATIONS. Handbook of National Accounting: Integrated Environmental and Economic Accounting. New York: EUROPENA COMISSION, 2003. 598p.

UNITED NATIONS. Sendai Framework for Disaster Risk Reduction 2015-2030. SENDAI: United Nations, 2015. 37p.

VALENCIO, N. Desastres: tecnicismo e sofrimento social. Ciência \& Saúde Coletiva, v. 19, n. 9, p. 3631-3644, 2014.

VEIGA, J.E. Desenvolvimento sustentável: o desafio do século XXI. Rio de Janeiro: Ed. Garamond, 2005.

VENTURATO-LANDMAM, R.; VALENCIO, N. “A alagação ofende!": considerações sociológicas acerca de um desastre silente no Alto Juruá, Acre, Brasil. Novos Cadernos NAEA, v. 17, n. 2, p. 239-264, dez. 2014.

VIVIEN, F. D. Le développement soutenable. Paris: La Découverte, 2005.

WESENER, A. Temporary urbanism and urban sustainability after a natural disaster: transitional community-initiated open spaces in Christchurch, New Zealand. Journal of Urbanism, n. 1072954, p. 1-17. 14 jul. 2015.

WORLD BANK. Natural hazards, unnatural disasters: the economics of effective prevention. Washington, D.C: World Bank Clearance Center Incorporation, 2010. 254p. 